\title{
Plataformas virtuales y fomento del aprendizaje colaborativo en estudiantes de Educación Superior*
}

Virtual platforms and promotion of collaborative learning in high education students.

Edgar René Zuña Macancela**

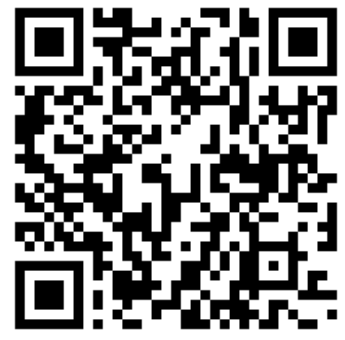

Wilson Javier Romero Berrones ${ }^{* * *}$

Julio César Palma Vidal ${ }^{* * * *}$

César Jorge Soledispa Baque ${ }^{* * * * *}$

\section{Resumen}

El aprendizaje colaborativo es un enfoque educativo constructivista que organiza el trabajo escolar dentro del aula para convertirla en una práctica social, las plataformas virtuales son idóneas para fomentar la colaboración en el contexto educativo, esta herramienta tecnológica logra esta dicha orientación, y al mismo tiempo realiza una reflexión sobre las posibles contribuciones en los procesos de

Artículo original derivado del proyecto de investigación titulado "Educación virtual". Entidad financiadora: Autores, fecha de realización entre 2019.

** Master en Educación. Universidad Agraria del Ecuador. Guayaquil, Ecuador. E-mail: ezuna@uagraria.edu.ec.

ORCID:

http://orcid.org/0000-0003-3730-0181.

*** Master en Educación. Universidad Agraria del Ecuador. Guayaquil, Ecuador. E-mail: wromero@uagrairia.edu.ec. ORCID: http://orcid.org/0000-0003-45696024.

**** Master en Educación. Universidad Agraria del Ecuador. Guayaquil, Ecuador. E-mail: japalmav@gmail.com. ORCID: http://orcid.org/0000-0001-9407-5721.

${ }^{* * * * *}$ Master en Educación. Unidad Educativa Ancón Bachillerato Internacional. Guayaquil, Ecuador. E-mail: cesarjorge1@hotmail.com. ORCID: http://orcid.org/ 0000-0002-9316-1262.

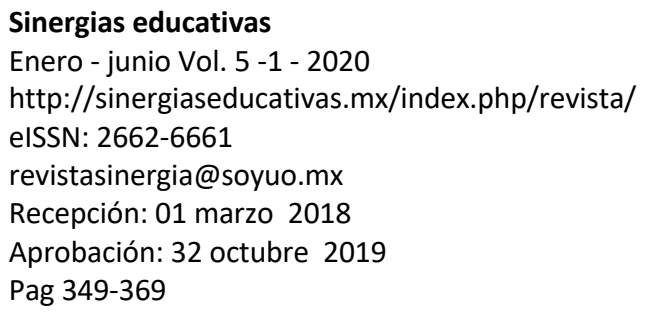

Disponible en

http://www.redalyc.org/articulo.oa?id=57356143200 27

Atribución/Reconocimiento-NoComercialCompartirlgual 4.0 Licencia Pública Internacional CC BY-NC-SA 4.0

https://creativecommons.org/licenses/by-ncsa/4.0/legalcode.es 
enseñanza, así mismo el demostrar que es un conjunto de estrategias y técnicas que propician el progreso de experiencias personales y sociales, donde cada integrante del grupo es responsable de su propio desarrollo cognitivo con ayuda de los demás miembros del grupo. El estudio, trata de explicar brevemente los antecedentes de las plataformas virtuales, y el aprendizaje colaborativo, la fundamentación teórica, los referentes pedagógicos, las estrategias metodológicas que se aplican en el aula y el método de investigación que se aplica en el artículo, investigación cualitativa, las técnicas para la recolección de los datos, además se investigó el uso de Edmodo como un entorno virtual de aprendizaje para la asignatura ofimática en el segundo Año de educación superior de la Universidad Agraria.

Palabras clave: plataformas virtuales, Edmodo, Aprendizaje colaborativo.

\begin{abstract}
Collaborative learning is a constructivist educational approach that organizes school work within the classroom to turn it into a social practice, virtual platforms are ideal to foster collaboration in the educational context, this technological tool achieves this orientation, and at the same time performs a reflection on the possible contributions in the teaching processes, as well as demonstrating that it is a set of strategies and techniques that promote the progress of personal and social experiences, where each group member is responsible for their own cognitive development with the help of Other group members. The study tries to explain briefly the background of virtual platforms, and collaborative learning, theoretical foundation, pedagogical references, methodological strategies that are applied in the classroom and the research method that is applied in the article, qualitative research, the techniques for data collection, the use of Edmodo as a virtual learning environment for the office automation in the second year of higher education at the Agrarian University was also investigated.
\end{abstract}

Key words: virtual platforms, collaborative learning, Constructivism, virtual classroom. 


\section{Introducción}

En la educación superior cada vez aparecen nuevas plataformas virtuales educativas más sofisticadas y con nuevas herramientas, que surgen como el remedio para resolver los problemas y los fracasos del estudiante universitario y la poca formación de los docentes, las plataformas virtuales ofrecen una formación personalizada $\mathrm{y}$ centrada en el estudiante, optimando los procesos de enseñanza y eliminando algunas dificultades como el tiempo y el espacio de la enseñanza presencial y tradicional, puesto que las plataformas virtuales crean un enfoque constructivista, como el aprendizaje colaborativo entre docentes y alumnos.

Autores como Fernández y Valverde (2013), afirman "que los entornos virtuales de aprendizaje ofrecen una serie de posibilidades para procesos de colaboración, donde el alumnado produce conocimiento de forma activa, formulando ideas que son compartidas y construidas a partir de las reacciones y respuestas de los demás" (p.98)

El nuevo paradigma educativo rompe una barrera caduca y crea un nuevo rol para la figura del profesor, pues deja de ser por un lado el actor principal y por otro sus actividades cambian de ser directivas a ser orientadoras y mediadoras, cuya función principal cambia de ser instructor a ser promotor de ambientes de aprendizaje.

En esta figura existe un universo real que experimentamos, el significado es impuesto por el universo, para Gross "se entiende la enseñanza como un proceso que no se centra en la transmisión de la información al alumno, sino que debe focalizarse en el desarrollo de habilidades para construir y reconstruir conocimientos en respuesta a la demanda de un determinado contexto o situación" (p.1).

Los recursos pedagógicos diseñados en una plataforma virtual representan una destreza innovadora que facilita la interacción y la cooperación porque promueven el aprendizaje simultáneo y colaborativo a pesar de las limitaciones de la distancia y la estabilidad, que se han convertido cada vez más en una oportunidad de habilidad individual en situaciones específicas. Es por ello que los resultados de este estudio generan una reflexión de la práctica 
educativa en el uso de estrategias de las plataformas virtuales, considerando que estos entornos educativos no se crean de manera automática y natural, sino que han de crearse como parte de los ambientes de aprendizaje, y para ello es importante identificar el uso común de estrategias de aprendizaje y las preferencias y estilos que el joven emplea para el aprendizaje colaborativo en su acercamiento a las plataformas virtuales.

El objetivo de este artículo consistió en implantar un modelo de plataforma virtual de enseñanza establecidas hoy en día a nivel mundial muy reconocida y muy exitosas como son Moodle y Edmodo por sus múltiples virtualidades como generar el conocimiento y la evaluación, así mismo de sus excelentes las características que poseen de las tácticas de aprendizaje utilizadas por los jóvenes de nivel de educación superior, así como su acercamiento al aprendizaje colaborativo en entornos virtuales.

Las plataformas virtuales son un conjunto herramientas tecnologías fundamentales que sirven para el desarrollo del aprendizaje y la enseñanza del alumno de manera individual y social, de la misma manera una herramienta virtual que sirve para la interacción activa entre el profesor y el alumno, el docente a través de las guía y apoyo constantes crea recursos pedagógicos de manera sincrónica y asincrónica y la comparte en la nube como son los wikis, Chat, videos conferencias, evaluación online, tareas, de la misma manera el alumnos recepta e investiga, realiza trabajo colaborativo, trabajo grupal e individual, además de reflexionar y construir su propio conocimiento.

Las plataformas virtuales como una opción de aprendizaje colaborativo

Según Rojas et al. (2014), establece que "Las plataformas virtuales de formación, si se realizan en ellas buenas prácticas, aumentan la motivación del alumnado a la hora de participar en las actividades y son un buen recurso para lograr que los participantes incrementen su competencia para poder llevar a cabo, al término de su formación, acciones de tipo preventivo" (p.239) 
De acuerdo con el autor en su estudio publicado, relaciona a las plataformas virtuales como la medicina del enfermo perfectas para desarrollar cambios significativos con un enfoque constructivista y social, así mismo las plataforma virtuales fomentan la colaboración y la comunicación, crea conocimiento, gestiona y comparte recurso, fomenta el trabajo en equipo, en fin enseñar en plataforma virtuales es una enseñanza cualitativamente mejor e innovadora que los planteados por paradigmas tradicionales.

En la educación superior las plataformas virtuales han alcanzado un progreso tecnológicos y científico muy significativo que han obligado a los directivos de instituciones universitarias, docentes, y muchas de las veces los estudiantes que han tenido que capacitarse $\mathrm{y}$ adaptarse a los constante cambios que se genera a diarios; las plataforma educativas han proporcionado diversas herramientas muy útil para la educación que sirven para planificar, desarrollar y optimizar los procesos de enseñanza y aprendizaje, de tal manera que se promueva la participación colaborativa de forma sincrónica y asincrónica entre los profesores y los estudiantes, y entre los propios docentes.

A partir de este escenario es urgente que los actores educativos se centren en la construcción de ambientes de aprendizaje que les permitan desarrollar tales requerimientos, en este contenido, las herramientas tecnológicas proporcionan el beneficio de ir acorde y al ritmo del desarrollo del mundo globalizado, por lo que el desfase en épocas deja de ser un riesgo latente, y por otra parte, proporcionan la organización de la información digital al alcance y con ello la posibilidad de la interacción con el mundo, eliminando los contratiempos que la distancia contrapone al contacto presencial; en el ámbito del aprendizaje abre la ventana a un nuevo formato en el que la autonomía y la autodirección se convierten en la forma idónea para administrar el proceso cognitivo.

Sin embargo, este camino no viene trazado desde la tecnología en sí, sino que para que sea una herramienta pedagógica, su diseño debe estar respaldado en las teorías del aprendizaje que de alguna forma retoman la dimensión de lo virtual. Breansford, "señala entre los principales aspectos para que se generen estos nuevos ambientes de aprendizaje, la necesidad de promover cambios en las metas 
educativas, y entender que los ambientes deberán ir centrados en quien aprende y no en quien enseña, en el proceso de adquisición y no en la permanencia del conocimiento, y en el terreno de la evaluación como la valoración de los mecanismos y la retroalimentación, y no en el producto, ello implica ver la educación como un proceso de formación y no de información, en la conformación de comunidades de aprendizaje basados en la socialización mediatizada del saber, en lugar de la conducción lineal centrada en el que más sabe".

La integración de estas nuevas perspectivas requiere de toda una sistematización que tome en cuenta la innovación de los medios digitales, de esta manera, la interacción mediada en los medios virtuales mediante las bondades de los alcances tecnológicos representa una oportunidad. Un entorno virtual es un ambiente de aprendizaje basado en medios digitales donde la interacción adquiere diferentes matices, ya que puede ser síncrono o asíncrono, es un todo organizado para que confluyan en su uso un conjunto de sujetos en la construcción de los saberes.

Establece que los entornos virtuales para el aprendizaje "aulas sin paredes" y afirma que es un espacio social virtual, cuyo mejor exponente actual es Internet, no es presencial, sino representacional, no es proximal, sino distal, no es sincrónico, sino metacrónico, y no se basa en recintos espaciales con interior, frontera y exterior, sino que depende de redes electrónicas cuyos nodos de interacción pueden estar diseminados por diversos países.

Edmodo como una plataforma de aprendizaje

Según Díaz Pinzón (2017), Edmodo permite establecer un espacio virtual de comunicación con los estudiantes y docentes, en el que se pueden hacer comentarios y aportes de las actividades realizadas, adjuntar archivos y enlaces, establecer un calendario de trabajo, así como de actividades, evaluaciones y gestionarlas" (p.9).

La plataforma educativa Edmodo propicia un espacio en la nube que sirve de apoyo para la construcción de conocimiento que se genera a partir de las contribuciones individuales y grupales de los participantes, así mismo permite categorizar las aportaciones 
realizadas durante el proceso de aprendizaje con objeto de facilitar la reflexión sobre el contenido de la propia intervención, además permite crear un espacio virtual de comunicación con los estudiantes y docentes, Edmodo es la herramienta que los profesores necesitan para compartir las clases, enviar mensajes, archivos y enlaces de un video de YouTube o link de alguna guía, un calendario de trabajo, así como proponer tareas, evaluaciones y actividades accesible en cualquier lugar.

Según el sitio web oficial de Edmodo. Visible body: Edmodo. Mariners Island Blvd, San Mateo.: Argosy Publishing. Recuperado de https://new.edmodo.com/ "Edmodo es una red educativa global que ayuda a conectar a todos los estudiantes con la gente y recursos necesarios para desarrollar todo su potencial”.

Procedimiento de trabajo con la plataforma Edmodo

Edmodo tiene tres tipos módulos, y cada uno de ellos puede utilizar la red de distinta forma. Profesor, estudiante y Familiar.

Desde la experiencia didáctica en la Universidad Agraria, una plataforma virtual ofrece recursos tecnológicos a toda la comunidad educativa para realizar las diferentes etapas del proceso de enseñanza y el aprendizaje, planificación de una clase, evaluación de una clase o del currículo.

En la Universidad Agraria, después de realizar un análisis de varias plataforma virtuales del medio y nivel mundial, así mismo se hizo comparaciones con múltiples entornos virtuales, se seleccionó la plataforma Edmodo por ser muy práctico y sencilla en su uso, además de ser open source y una de las más prestigiosa a nivel mundial, este entorno educativo se la usará para educación a distancia y para concluir modulo o curso de alguna asignatura que estén pendientes por el alumno, de esta forma la Plataforma Edmodo nos permite aprender días siguientes maneras:

Edmodo dispone de múltiples herramientas tecnológicas como son:

Comunicación asincrónica, El chat, asincrónica el correo electrónico $\mathrm{y}$ foros 
Para la gestión de materiales de aprendizaje: Contenido de las clases, de los cursos, ejercicios, las prácticas, etc.

Para la gestión de los participantes: Agenda, Mensajes, anuncios, etc.

Crear una cuenta profesor: Llenar un formulario donde nos piden usuario, contraseña y cuenta de e-mail, Una vez creada la cuenta, accedemos a la plataforma (de aspecto similar a una red social como Facebook) donde podemos crear un grupo, por ejemplo, para nuestra aula. Una vez creado el grupo nos envían un código exclusivo para ese grupo, este código servirá para que nuestros alumnos soliciten entrar en este grupo con el perfil del estudiante, creando un usuario, contraseña, y poniendo su nombre y apellido (no hace falta cuenta de e-mail). Cada usuario puede elegir una imagen o subir una foto dentro de Preferencias.

Una vez registrado todo el grupo, cada alumno-usuario puede mandar mensajes al grupo o al profesor. Con nuestro perfil de profesor además podemos enviar al grupo encuestas o asignaciones (tareas o actividades que deben remitirnos antes de una fecha determinada). Una vez que los alumnos realicen la actividad nos la envían a través de la plataforma y podemos calificarla.

Crear cuenta estudiante: El alumnado que accede dispone de una cuenta propia que le permite establecer un dialogo con sus profesores, manteniendo su información totalmente ordenada, registrada y segura ya que los estudiantes ni siquiera necesitan cuenta de correo, solo es necesario un código que el profesorado le proporciona. También les permite la creación de un calendario donde se van programando todas las tareas académicas, para que todos los usuarios-padres, madres, profesorado y alumnado puedan consultarlo siempre que lo necesiten. Entre las características más destacadas se encuentran:

Crear cuenta estudiante: Llenar un formulario con tu número de teléfono, correo electrónico para Obtener la información que necesitas para apoyar el aprendizaje de tu hijo en casa junto con los recursos que necesitan para tener éxito en la escuela. 
Para lograr un éxito en el estudio del objeto aprendizaje colaborativo es necesario crear los objetivos y la pregunta de la investigación

¿Cómo incide el uso de las plataformas virtuales en el fomento del aprendizaje colaborativo en los estudiantes de la Universidad Agraria Del Ecuador?

Analizar la incidencia del uso de las plataformas virtuales para fomentar aprendizaje colaborativo de los estudiantes del segundo Año, realizando una investigación cualitativa en la Universidad Agraria del Ecuador para diseñar un aula virtual de aprendizaje en Edmodo

1. Establecer el tipo de herramientas de plataformas virtuales que proporcionan el aprendizaje colaborativo en el segundo año a través de encuesta a estudiantes.

2. Diseñar el curso en la asignatura de los tics en la plataforma virtual LMS Edmodo

3. Determinar las actividades del curso que propician el trabajo colaborativo.

Los métodos tradicionales de enseñanza superior universitaria, acompañado del poco uso de las herramientas Tecnológicas en las aulas de clase, provocan que los estudiantes del segundo Año de la Universidad Agraria del Ecuador presenten inconvenientes en el aprendizaje de varias asignaturas, afectando su rendimiento académico y el desarrollo de sus habilidades y destrezas para su formación profesional. Porque solo utilizan libros, pizarra, entrevistas y debates, no usan una herramienta de apoyo como Plataformas Virtuales, las clases se vuelven monótonas y hay mucha desmotivación, el pensamiento de los estudiantes no conjugan el interés de aprender y discernir, al utilizar una Plataforma Virtual en la Carrera de Informática, se fomenta la colaboración en grupos y sería el aporte factible por cuanto garantizaría de mejor manera los aprendizajes.

Para este elemento negativo es conveniente realizar un cambio sustancial en el aspecto académico que plantea la presente 
investigación. Las apreciaciones que se formulan en cada una de las estructuras de la tesis, están orientadas a cambiar una estructura curricular que ya no responde a las exigencias de la época actual y a los avances de las ciencias tecnológicas que día a día aportan con contenidos curriculares que se los debe aplicar en la vida práctica de los futuros ingenieros en el área de la informática.

Con la implementación de una Plataforma Virtual Edmodo en la Carrera de informática, se logrará beneficios, por cuanto se aprovecha las herramientas tecnológicas como, foros, wikis, correos electrónicos, chat, multimedia y funcionará en línea con dominio de IP pública www.newedomodo.com y ayudará a los profesores a educar con un nueva herramienta metodológicas innovadora e interactiva, en una educación social constructivista basado en el trabajo colaborativo, llenará un vacío que hasta ahora ha resultado difícil de superar, pues anteriormente no estaban disponibles los recursos tecnológicos que al principio fue muy selectiva y costoso para su implementación, unido esto y por supuesto a la falta de formación de docentes en este ámbito tecnológico para que puedan enfrentar estos retos.

En la Universidad Agraria del Ecuador no existe una investigación formal sobre el uso de las plataformas virtuales para el uso del aprendizaje colaborativo por parte de los maestros en el aula de clase, las experiencias de estas, en otros países, son muy exitosas, producto de ello, los estudiantes de educación del segundo Año, en la facultad de informática podrán:

ü Aumentar el interés por el módulo estudiado

ü Mejorar la capacidad de aprendizaje para resolver problemas

ü Elevar su nivel de confianza en sí mismo

ü Incrementar la creatividad, destreza y la imaginación

ü Permitirán una mayor integración e interacción

ü Trabajar en grupo 
Otra de las razones para el uso de las plataformas virtuales es los contenidos curriculares que puedes trabajar en sus diferentes herramientas que poseen: como es plan anual, plan de bloque y plan semanal ya que permiten presentar la información de una manera muy distinta a como lo hacían los tradicionales libros. Pues se tratan de contenidos más dinámicos con una característica fundamental: la interactividad, Las wikis. Foro. Chat. Ellos fomentan una actitud activa del estudiante frente al carácter de exposición o pasivo, lo que hace posible una mayor implicación del estudiante en su formación.

Con este aporte para los docentes y estudiantes de la facultad de informática, tendrá un impacto muy significativo porque tienen la posibilidad de crear contenidos educativos en línea a través de la plataforma con los intereses o las particularidades de cada estudiante, también todo el personal están involucrado a utilizar la nueva herramienta virtual, además mantener contacto con comunidades educativas a través de la web, donde se muestran proyectos educativos de todo nivel, adaptables a la realidad de la institución, para mejorar el proceso de aprendizaje.

\section{Materiales y métodos}

Unas de las razones para realizar el estudio es la profundidad del caso aprendizaje colaborativo a través de las plataformas virtuales de estudiantes de la Universidad Agraria, la investigación es de tipo cualitativa que busca analizar las características de la realidad en los contextos educativos, asimismo se aplica la modalidad interactiva "La investigación cualitativa está más preocupada en la comprensión de los fenómenos sociales desde la perspectiva de los participantes" (McMilan y Shumacher, 2005, p.19).

Otro método que se plantea en este estudio es la etnografía como un complemento a metodológica, pues integra un paradigma cualitativo, interpretando los datos de un cuestionario, aplicado a un grupo social o cultural "La etnografía se refiere al trabajo, el proceso o la forma de investigación que nos permite realizar un estudio descriptivo y un análisis teóricamente orientado de una cultura o de algunos aspectos concretos de una cultura" (Serra, 2003, p.165) 
Así mismo se emplea en este artículo la Técnica interactiva que radica en un estudio de análisis profundo mediante el empleo de técnicas para recoger los datos de los fenómenos en sus escenas naturales. Afirman: que Las Técnicas Interactivas para la Investigación Social Cualitativa reconocen como marcos de referencia y de orientación los intereses de ubicar, orientar e interpretar; de emancipar y liberar y de reconstruir y resinificar, los cuales marcan una identidad, una intencionalidad, una postura frente a la investigación, al conocimiento, y a los sujetos participantes de la investigación; a partir de estos intereses las técnicas interactivas promueven procesos que buscan el cambio y la re significación de las prácticas sociales así como el reconocimiento de los sujetos. (p.34)

El plan se centra en estudiantes del segundo año de educación superior, estudiantes cuyas edades oscilan entre los 20 y 22 años de edad.

Para la muestra se tomaron en cuenta al grupo de estudiante del paralelo "B" del segundo Año de la carrea de informática facultad de computación, a este grupo de jóvenes se les aplicó la encuesta

Para la recolección de datos se diseñó 1 cuestionarios, La muestra está representada en los estudiantes segundo año de la carrera de informática a quienes se les aplico la encuesta, 15 preguntas cerradas basada en las variables de estudio, las plataformas virtuales para fomentar el aprendizaje colaborativo de forma presencial, respuestas largas partir de dos variables.

Las encuestas se aplicarán al inicio y al finalizar la investigación; está organizada en tres etapas, la inicial un diagnóstico, la segunda de diseño y la tercera de verificación.

En la fase inicial la encuesta se aplica a 35 estudiantes para diagnosticar los conocimientos sobre tecnología plataformas virtuales y otra sección sobre el uso pedagógico de las aulas virtuales que fomentan aprendizaje colaborativo. (Ver tabla 1)

En la segunda etapa se crea el grupo Curso: Segundo año, clase Ofimática en la plataforma Edmodo, los estudiantes inscrito por el 
profesor o interesados en participar en el aula virtual, deben suscribirse con un código que la plataforma les asigna. Posteriormente ya registrados encontrarán las actividades a desarrollar en la plataforma, además de material de consulta para la asignatura Ofimática el grupo está conformado por 35 estudiantes.

El Gráfico muestra la creación del grupo, servirá para crear una clase con u código para que el alumno se suscriba y realice las tareas diseñada por el profesor.

El Gráfico muestra la creación de los contenidos curriculares, servirá que servirá como guía para que el alumno y se suscriba con un código.

Muestra la actividad de forma vacía es decir sin ningún estudiante que haya intervenido en el proceso de interacción con la entrega de algún trabajo.

Según López (1998) afirma: La encuesta se ha convertido En una herramienta fundamental para el estudio de las relaciones sociales. Las organizaciones contemporáneas, políticas, económicas $\mathrm{o}$ sociales, utilizan esta técnica como un instrumento indispensable para conocer el comportamiento de sus grupos de interés y tomar decisiones sobre ellos. (p.1)

El cuestionario no lleva ninguna identificación de los estudiantes a quienes se les aplicó, porque esos datos no nos interesan. La encuesta es una técnica que se puede aplicar a sectores más amplios del universo, es menos costosa que cualquier otra técnica.

Se realizó una encuesta el 16 de julio de 2019, a 35 estudiantes de segundo año, de la carrera informática de la Universidad Agraria, de forma presencia de acuerdo a las dos variables, plataformas virtuales y aprendizaje colaborativo, ver anexo 2. Los acuerdos utilizados fueron E: Estudiante, P: Pregunta, como los estudiantes encuestados fueron 35 la tabla 1, y las preguntas desde Pregunta 1 hasta Pregunta 10, y la opción de respuestas son SI $(\mathrm{S})$ y NO $(\mathrm{N})$

P1. Me gustaría que los profesores utilicen otros métodos de aprendizajes donde tengas mayor participación 
P2. Las tareas colaborativas me ayudan a entender y complementar los trabajos en equipo

P3. Cuando trabajo en grupo considero que aprendo más y me ayudan a interactuar con mis compañeros

P.4 Los trabajos en las diferentes asignaturas lo realizan de manera frecuente en grupo

P.5 Considero que el bajo rendimiento del estudiante, un alto porcentaje tiene que ver con los métodos que utilizan los docentes al impartir su materia

P.6 Me gustaría que se implementen nuevos modelos de aprendizaje utilizando las Tics

P.7 Me gustaría que se aplique el aprendizaje de tus asignaturas utilizando los recursos que ofrecen las TICS

P.8 Te considera experto en el manejo de la computadora

P.9. Manejas correctamente el internet y los diferentes navegadores web

P.10. Utilizando Plataformas virtuales ha mejorado su rendimiento académico

\section{Resultados}

Tabla 1 Esta dirigida a los estudiantes de la Universidad Agraria del Ecuador

\begin{tabular}{l|l|l|l|l|l} 
No & Preguntas & $\mathrm{Si}$ & $\%$ & No & $\%$ \\
\hline 1 & $\begin{array}{l}\text { Me gustaría que los profesores utilicen otros } \\
\text { métodos de aprendizajes donde tengas mayor } \\
\text { participación }\end{array}$ & 28 & 80 & 7 & 20 \\
\hline 2 & $\begin{array}{l}\text { Las tareas colaborativas me ayudan a } \\
\text { entender y complementar los trabajos en } \\
\text { equipo }\end{array}$ & 21 & 60 & 14 & 40
\end{tabular}




\begin{tabular}{l|l|l|l|l|l}
3 & $\begin{array}{l}\text { Cuando trabajo en grupo considero que } \\
\text { aprendo más y me ayudan a interactuar con } \\
\text { mis compañeros }\end{array}$ & 26 & 74 & 9 & 26 \\
\hline 4 & $\begin{array}{l}\text { Los trabajos en las diferentes asignaturas lo } \\
\text { realizan de manera frecuente en grupo }\end{array}$ & 27 & 75 & 9 & 25 \\
\hline 5 & $\begin{array}{l}\text { Considero que el bajo rendimiento del } \\
\text { estudiante, un alto porcentaje tiene que ver } \\
\text { con los métodos que utilizan los docentes al } \\
\text { impartir su materia }\end{array}$ & 23 & 66 & 12 & 34 \\
\hline 6 & $\begin{array}{l}\text { Me gustaría que se implementen nuevos } \\
\text { modelos de aprendizaje utilizando las Tics }\end{array}$ & 30 & 86 & 5 & 14 \\
\hline 7 & $\begin{array}{l}\text { Me gustaría trabajar con una plataforma } \\
\text { virtual para el desarrollo de la asignatura } \\
\text { básica de mi pensum académico }\end{array}$ & 22 & 63 & 13 & 37 \\
\hline 8 & $\begin{array}{l}\text { Te considera experto en el manejo de la } \\
\text { computadora }\end{array}$ & 19 & 54 & 16 & 46 \\
\hline 9 & $\begin{array}{l}\text { Manejas correctamente el internet y los } \\
\text { diferentes navegadores web }\end{array}$ & 27 & 77 & 8 & 23 \\
\hline 10 & $\begin{array}{l}\text { Utilizando Plataformas virtuales ha mejorado } \\
\text { su rendimiento académico }\end{array}$ & 26 & 74 & 9 & 26 \\
\hline
\end{tabular}

Los autores

Pregunta 1: Se observa que el $80 \%$ de los estudiantes les gustaría que los profesores utilicen otros métodos de enseñanza, y un $20 \%$ no están de acuerdo porque les parecen difícil para su formación académica.

Pregunta 2: El 60\% de los estudiantes afirman que las tareas colaborativas les ayudan a entender los trabajos y un $40 \%$ se les hace difícil trabajar con esa metodología. Por lo consiguiente es factible que el alumno pueda ingresar a la plataforma Edmodo

Pregunta 3: Se observó en la tabla que el 74\% de los alumnos encuestados afirman que cuando trabajan en conjunto aprenden más e interactúan mejor con sus compañeros y un $26 \%$ consideran que es pérdida de tiempo que no se prende nada. De tal manera que un gran porcentaje de estudiantes sabe cómo trabajar en equipo $\mathrm{y}$ complementarse así mismo en un determinado tema

Pregunta 4: En el análisis de la interrogación cuatro el 75\% de la población encuestada están de acuerdo que su rendimiento 
académico ha mejorado trabajando en grupo usando herramientas virtuales, y un $25 \%$ declara lo contrario. Entonces, los resultados determinan, que los alumnos al trabajar en conjunto las actividades académicas tienen mayor significado y son muy útiles para ellos.

Pregunta 5: El 66\% de la población considera que el docente debe aplicar un enfoque pedagógico distinto al tradicional, y un 34\% cree lo contrario. De esta manera la encuestada determina que los estudiantes en algún momento han usado la tecnología para recibir clase, lo cual es importante aplicarla en la institución educativa

Pregunta 6: Este apartado los resultados de la encuesta el 86\% de estudiantes consideran que se debe implementar un nuevo modelo pedagógico interactivo, constructivista y social y el otro $14 \%$ prefiere las clases traiciónales. Con un nuevo modelo pedagógico interactivo e innovador, permite la autonomía en los estudiantes y la necesidad de manipular, navegar y compartir los recursos tecnológicos que ofrecen las herramientas tics.

Pregunta 7: Se observa que el 63\% de los alumnos afirman que debe implementar este método didáctico, porque al usar una plataforma virtual podría facilitar su aprendizaje y el 37 aseveran lo contrario. Un alto porcentaje de estudiantes considera que la tecnología mejoría sus habilidades sociales para comunicarse y para manejar de forma adecuada.

Pregunta 8: En la tabla se observa que el 54\% de los universitarios maneja el computador y programas de forma adecuada, y un $46 \%$ manejan lo básico para realizar las tareas en casa y en la clase. Con este análisis de esta pregunta un porcentaje elevado considera que el ordenador es necesario en su formación universitaria.

Pregunta 9: En este apartado se observa que el 73\% de la población estudiantil encuestada señala que maneja de forma correcta el internet y sus navegadores, y un $23 \%$ declara lo contrario. Así mismo los estudiantes afirman que utilizar internet ha sido muy beneficioso para sus estudios porque hay cantidades de sitios académicos para investigar y contactar información fidedigna, además de ser muy útil en su vida cotidiana. 
Pregunta 10: Se observó en este apartado que el $77 \%$ de los estudiantes universitarios afirman que su rendimiento académico ha mejorado cuando han utilizado plataformas virtuales como Edmodo, y un $23 \%$ revela lo contrario. De esta manera los estudiantes aseveran que utilizar entornos virtuales se siente más cómodos y amigables con el mundo tecnológico que los rodeas, así mismo se siente motivados y creen que han mejorado en sus prácticas para comunicarse y para manejar la tecnología.

En este apartado se visualiza de forma general que los resultados de la tabla de las 10 preguntas, exponen que las plataformas virtuales pueden usarse para reducir las horas presenciales sin afectar de carácter significativo la adquisición de conocimientos por parte de los universitarios.

Encuesta de satisfacción

Para la recolección de datos de la encuesta de satisfacción se diseñó un cuestionario de 5 preguntas, basada en la plataforma Edmodo, plataforma virtual para fomentar el aprendizaje colaborativo de forma presencial, con un rango de 1 a 5 respuestas en escala de tipo Likert a partir de dos variables de análisis, aplicada a 10 estudiante de segundo año de nivel superior de la Universidad Agraria el 1 de octubre de 2019, (ver tabla 2).

La escala de estimación es la siguiente

5 = Muy fácil

4 = Fácil

$3=$ Regular

$2=$ Difícil

$1=1$ Muy difícil

Según Matas (2018), estableces que "Las llamadas ,escalas Likert" son instrumentos psicométricos donde el encuestado debe indicar su 
acuerdo o desacuerdo sobre una afirmación, ítem o reactivo, lo que se realiza a través de una escala ordenada y unidimensional" (p.2)

Tabla 2 Resultados encuesta de satisfacción

\begin{tabular}{l|l|l|l|l|l} 
Aspecto & 1 & 2 & 3 & 4 & 5 \\
\hline $\begin{array}{l}\text { Registrarse en el grupo de la plataforma } \\
\text { Edmodo }\end{array}$ & & & & & 10 \\
\hline Acceder a links publicados en Edmodo & & & & 1 & 9 \\
\hline Encontrar la prueba de Ofimática & & & & & 10 \\
\hline Realizar la prueba de Ofimática & & & & 1 & 9 \\
\hline Ver el resultado de la prueba de ofimática & & & & & 10
\end{tabular}

Los autores

Pregunta 1: En este apartado se observa que el $100 \%$ de los estudiantes universitarios encuestados lograron una calificación de 5, al darse de alta en la plataforma de Edmodo, new.edmodo.com, Según los que refleja la tabla la escala cualitativa fue muy fácil el ingreso.

Pregunta 2: Se observó en la tabla que el $90 \%$ de los alumnos de La Universidad Agraria obtuvieron una calificación de 5 (Muy fácil), ingresar a links publicados en la plataforma Edmodo como videos, Chat, wikis documentos en PDF; y un 10\% califican con un 4 (Fácil), encontrar la misma dirección URL.

Pregunta 3: Según el análisis de los datos de la tabla se determina que el $100 \%$ de los universitarios califican con un 5(Muy fácil), encontrar en la plataforma virtual la prueba de ofimática.

Pregunta 4: Se visualiza en la tabla que el $90 \%$ de los alumnos calificaron con un 5 (Muy fácil), realizar la prueba de ofimática en la plataforma Edmodo. El 10\% de los encuestados evaluaron con un 4 (Fácil), realizar la prueba en la plataforma virtual.

Pregunta 5: Se observó en la tabla que el 100\% de los estudiantes obtuvieron una calificación de 5 (Muy fácil), ver el resultado de la prueba de ofimática, esta se muestra al terminar la prueba. 


\section{Conclusiones}

Las limitaciones que se evidencio en este estudio es que muchos docentes se resisten al cambio, de implementar las herramientas tecnología en el aula, aun siente el miedo y prefieren una educación tradicional, así mismo una limitada gestión económica por parte de quienes están en la dirección de la institución para la incorporación de estrategia de aprendizaje y satisfacción de los estudiantes, se origina la débil enseñanza y poco uso de las herramientas tics aliada a la educación, debido a que no se emplean de forma idónea acciones estratégicas orientas a consolidar a una educación de calidad, y por ende la participación de los estudiantes aún no está 100, en el fortalecimiento de las plataformas educativas.

En relación al análisis de los datos de las preguntas del cuestionario, queda en evidencia que los estudiantes acaban de conocer a las plataformas virtuales y se están familiarizando con ella, específicamente con Edmodo y por ende los resultados obtenidos van en conformidad con los sucesos descritos

Se pudo implantar en la Universidad Agraria Del Ecuador el uso de plataforma virtual Edmodo. Específicamente en la enseñanza de la Ofimática relacionado con el computador, internet y las plataformas virtuales. En forma general Las Tecnologías de Información y Comunicaciones (TIC) favorecieron un nuevo paradigma de aprendizaje, en donde se fomenta la colaboración en los espacios virtuales que propician interacciones sociales entre los participantes de este proceso educativo.

Se bosquejaron actividades de enseñanza y aprendizaje, usando la plataforma educativa Edmodo en la asignatura Ofimática.

Se realizó una evaluación a la plataforma Edmodo para conocer cuál era su aceptación, comprobando una gran aprobación por parte de los alumnos de segundo año de la Universidad Agraria. 


\section{Referencias}

Albert Gomez, M. J. (2007). La Investigación Educativa: Claves Teoricas (Primera Edicion ed.). (J. M. Cejudo, Ed.) Madrid, España: McGraw Hill.

Bernal , C. A. (2010). Metodologia de la Investigación (Tercera Edición ed.). (O. Fernández Palma, Ed.) Bogotá, Colombia: Pearson Education.

Buzon García, H. (2008). La incorpoarcion de las plataforma virtuales a la enseñanza. Revista Latinoamerica de Tecnologia Educativa, 4(1), 77-100

Díaz Pinzón, J. ( 2017 ). Edmodo como Herramienta Virtual de Aprendizaje. Innova Research Journal.2(10),9-16

Fernández, M. R., \& Valverde, J. (2013). Comunidades de práctica: un modelo de intervención desde el aprendizaje colaborativo en entornos naturales. Revista científica de Educomunicación, 42(21), $97-105$

License., G. G. Moodle. (2017). Acerca de Moodle. España: MediaWiki. Obtenido de https://docs.moodle.org/all/es/Acerca_de_Moodle

López Romo , H. (1998). La metodología de encuesta. Técnicas de investigación en sociedad, cultura y comunicación.2(1), 33-73.

Matas, A. (2018). Diseño del formato de escalas tipo Likert: un estado de la cuestión. Revista electrónica de educación educativa.20(1), 38-47

McMillan, J., \& Schumacher, S. (2005). Investigación Educativa (Vol. 5). Madrid, España: PEearson Addison Wesley.

Méndez Álvarez, C. E. (2006). Metodología: Diseño y desarrollo del proceso de investigación. Limusa, Bogotá: Limusa S.a de C.V. 
Rojas Machado, N., Pérez Clemente, F., Torres Milord, I., \& Peláez Gómez , E. (2014). Las aulas virtuales: una opción para el desarrollo de la Educación Médica. Edumelentro, 2(6), 231-247

Serra, C. (2003). Etnografía escolar, etnografía de la educación. Revista de Educación. 1(334), 165-176.

Sverdlick, \& Ingrid. (2012). Buscando la calidad eduactiva. Buenos Aires: Laboratorio de Políticas Públicas 